\title{
IoT for the Failure of Climate-Change Mitigation and Adaptation and IIoT as a Future Solution
}

\author{
Nesma Abd El-Mawla ${ }^{1, *}$, Mahmoud Badawy², Hesham Arafat ${ }^{2}$ \\ ${ }^{1}$ Department of Communications and Electronics, Nile Higher Institute for Engineering and Science, Mansoura, Egypt \\ ${ }^{2}$ Department of Computers and Systems, Faculty of engineering, Mansoura University, Mansoura, Egypt \\ *Corresponding author: nesma.abdelmawla@gmail.com
}

Received January 14, 2019; Revised February 16, 2019; Accepted March 21, 2019

\begin{abstract}
Day after day the world stuck more and more in wars, pollution and so many other risk that threaten the environment. With a population of more than 7.3 billion, the planet suffers from continuous damage from human activity. As a result of these human distortions, climate change is one of the most fatal challenges that face the world. Climate Change won't be stopped or slowed by a single action, but with the help of too many small contributions from different fields, it will have an impressive impact. Changing to electricity generation, manufacturing, and transportation generate most headlines, but the technology field can also play a critical role. The Internet of Things (IoT) in particular, has the potential to reduce greenhouse emissions and help slow the rise of global temperatures. IoT includes more than super brilliant new gadgets and smart widgets. It also influences the Earth's condition, from its available resources to its climate. In this paper we are showing that technology itself could be the tool will save the world if we take advantage of it. Environmental monitoring is a broad application for the Internet of Things (IoT). It involves everything from watching levels of ozone in a meat packing facility to watching national forests for smoke. These solutions are the first step toward creating a numerous connected infrastructures to support innovative services, better flexibility and efficiency. We also make a spot on Industrial Internet of Things (IIoT) and its challenges as the future is for it.
\end{abstract}

Keywords: IoT, climate, environmental, challenges, risks

Cite This Article: Nesma Abd El-Mawla, Mahmoud Badawy, and Hesham Arafat, "IoT for the Failure of Climate-Change Mitigation and Adaptation and IIoT as a Future Solution." World Journal of Environmental Engineering, vol. 6, no. 1 (2019): 7-16. doi: 10.12691/wjee-6-1-2.

\section{Introduction}

Climate change represents a serious problem for people all around the world. However, the Internet of Things (IoT) offers several resources and variant tools that help businesses and governments reduce the bad effect of human activity on Earth. Climate change also fuels debates in large part because it inspires people to think in terms of scarcity. If they admit the existence of the problem, they might have to give up their luxuries and what they enjoy to reduce their carbon footprints. However, this isn't always the case. What is mainly causing the climate to warm, and weather to go wild, is flooding the atmosphere with carbon dioxide by humankind. We generate $\mathrm{CO} 2$ when we burn fuels such as petroleum, coal, or natural gas. We thus need both to become radically more energy-efficient and to drive carbon out of the electricity generating system, even as we use electricity in more ways (like powering vehicles). For the future, electricity must be produced with the help of sustainable sources such as solar and wind that don't create CO2. IoT can help in increasing the speed, power, and efficiency of consumer goods while still reducing energy consumption.

"The Internet of Things can help decarbonizes our energy system, provide modern energy systems to every human being, manage our infrastructure, and allow us to adapt to and address climate change." - Arun Majumdar, director of Stanford's Precourt Center

Since 2016 and till now, IoT advancements allowed companies, governments, and consumers to reverse climate change without the need of sacrificing their convenience. It's predicted that more consumers will participate in activities that allow businesses and governments to collect Big Data and to analyze it for the benefit of the environment. Connected devices pave the way to climate change and environmental health terms to be the future. In 2016, Gartner [1] research presented that smart cities around the world will use 1.6 billion connected devices. Also, a recent report by Ericsson claims that information and communication technologies (ICT) could help cut up to 63.5 Gtonnes of Greenhouse Gas (GHG) emissions by 2030 as shown in Figure 1. Furthermore, given the growing number of synergies the IoT creates between different industries, we could see for the first time a simultaneous reduction of industries' 
environmental footprint, thanks to energy savings and smarter solutions.

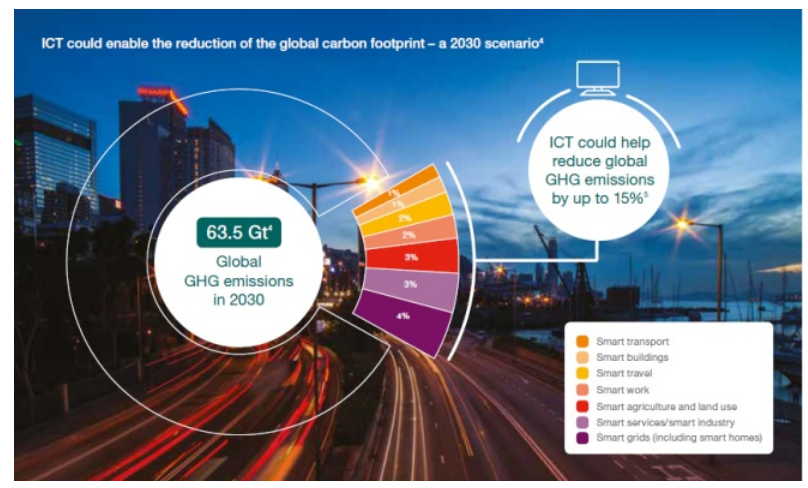

Figure 1. ICT reducing global GHG by 2030 (Source: Ericsson: ICT and the Low Carbon Economy)

The fact that all those industries and leaders around the world have the incentive to increase the adoption of IoT solutions, if they want to keep growing and stay competitive. That's why the Internet of Things may well be considered a powerful tool in the fight against climate change, precisely at a time when global leaders advocate for more economically viable and scalable ways to protect our planet. The world soon will be consisted of numerous connected systems which will make it possible to depend much more on variable sources of power like solar and wind. Many experts around the world thought such sustainable sources couldn't comprise more than 10\% of total energy production, at best, because of how intermittent they were, but as the potential of the IoT has become clearer, it now appears that as much as $80 \%$ of the world's total energy production could eventually come from renewable. For Example, NRG, the giant commercial utility works hard to achieve goal of reducing its overall carbon emissions $50 \%$ by 2030 , even as the company expects to continue growing and by 2050 it make a promise to cut $\mathrm{CO}_{2}$ an impressive $90 \%$, using emissions of 2014 as a baseline.

World leaders thinks the process of facing climate change is too complicated challenge, but estimates and evaluations by communications equipment giant Ericsson and environmental group Carbon War Room say the progress of machine-to-machine communications, or IoT, can get us a good part of the way there, reducing to as much as an $18 \%$ by 2030 [2]. IoT paves the way toward the creation of a smart electric system in which there can be great flexibility in both supply and demand. Also, a responsive energy network in which both production and usage can be quantified in real time, and correlated by the help of IoT smart systems. Power will no longer need to be produced only when it is consumed, because we will have new ways of predicting demand, usage, and storing energy. So, we could get to the truth proving that all of these massive numbers of connected devices will offer different additional ways to make humans, environment and its energy use more efficient, and reduce carbon production. It's so clear that IoT will not change our lives only but changing the entire planet.

"Technology is one of the few industries where new products tend to get better and use less power."

- Tim Mohin, AMD

\section{Global Risks 2019 Report \& Failure of Climate Change Mitigation and Adaptation}

The Global Risks report of 2019 in its 14th edition which nearly made by 1,000 of best decision-makers from the public and private sectors and civil society presents the top ten urgent risks facing the world. Nine out of ten respondents expect that economic and political confrontations will get worse between major powers this year. Over a ten-year, climate-change and weather policy failures are seen as the gravest threats. This year report presents an amazing detailed series of "what-if" topics discussing about future and examining many urgent points such as weather manipulation, emotionally responsive artificial intelligence, monetary populism, and other potential risks. On the human causes and effects of global risks chapter the theme of emotions is addressed obviously to call for bigger and more effective actions around rising levels of psychological strain all over the world $[3,4]$.

Table 1. Top 10 Global Risks In 2019

\begin{tabular}{|c|c|c|}
\hline & $\begin{array}{c}\text { Top 10 risks in terms of } \\
\text { Likelihood }\end{array}$ & Top 10 risks in terms of IMPACT \\
\hline 1 & Extreme weather events & Weapons of mass destruction \\
\hline 2 & $\begin{array}{c}\text { Failure of climate-change } \\
\text { mitigation and adaptation }\end{array}$ & $\begin{array}{c}\text { Failure of climate-change } \\
\text { mitigation and adaptation }\end{array}$ \\
\hline 3 & Natural disasters & Extreme weather events \\
\hline 4 & Data fraud or theft & Water crises \\
\hline 5 & Cyber-attacks & Natural disasters \\
\hline 6 & $\begin{array}{c}\text { Man-made environmental } \\
\text { disasters }\end{array}$ & $\begin{array}{c}\text { Biodiversity loss and ecosystem } \\
\text { collapse }\end{array}$ \\
\hline 7 & $\begin{array}{c}\text { Large-scale involuntary } \\
\text { migration }\end{array}$ & $\begin{array}{c}\text { Ciodiversity loss and } \\
\text { ecosystem collapse }\end{array}$ \\
\hline 8 & Water crises & Man-made environmental disasters \\
\hline 10 & $\begin{array}{c}\text { Asset bubbles in a major } \\
\text { economy }\end{array}$ & Spread of infectious diseases \\
\hline
\end{tabular}

Source: World Economic Forum Global Risks Perception Survey 2018-2019.

The two lists above are unlike, but there are still some common themes that connect the two. In the table above we can notice that environmental concerns accounted for three of the top five risks by likelihood and four by impact classification. With keen eyes we could also notice that "Failure of climate change mitigation and adaptation" takes the second place on both impact and likelihood lists as shown in Figure 2, reflecting respondents increasing concerns about environmental policy failure. Global economy, societal and geopolitical risks are also taking big concerns in terms of likelihood and impact. By increasing the human needs and depending on technology more and more, many new risks have been added to the world risks list and the surprise is that cyber-attacks appears in both top 10s, at number five for likelihood and seven for impact, while data fraud is at number four for likelihood only, showing that the technology is also involved in threaten the world safety and open a new issue about world security as we will show by the end of this paper.

Last year saw massive data breaches, with millions - if not billions - of people's data affected, as well as continued cyber-attacks on both public and private institutions and businesses. Most of respondents (82\%) 
expected that the danger of cyber-attacks will lead to theft of money and data to increase in 2019, with $80 \%$ believing they would disrupt operations.

"Reflects how new instabilities are being caused by the deepening integration of digital technologies into every aspect of life"

\section{- Survey says.}

Under "a significant decline in the available quality and quantity of fresh water, resulting in harmful effects on human health and-or economic activity” water crises, societal risk was defined and take a great priority on both lists. It is located at fourth place for impact and ninth for likelihood. The report warns of the macro-economic risks the world faces as we head into 2019.Respondents also expect increasing risks this year, related to "economic confrontations between major powers" (91\%) and "erosion of multilateral trading rules and agreements" with percent equals (88\%). Financial market volatility and speed world growth through 2018 square measure highlighted by the authors and so by the most recent International fund forecasts. As a result, the danger of associate degree quality bubble in a very major economy is tenth possibly. These risks don't exist in isolation or on separate. They're totally interconnected and each has the potential to affect the others as the following chart from the report explores.

\section{IoT Fights Climate Change}

One of the ways of achieving this task of fighting climate change is through advances in machine-to-machine communications, or the Internet of Things (IoT), which can have a big contribution to as much as an $18 \%$ reduction by 2030 [5]. IoT can be defined as a network of objects embedded with numerous number of sensors, software/application, network connectivity and computer capability, that can connect, manipulate and exchange data over the internet and enable smart solutions. IoT is already offering unique opportunities for addressing many environmental issues like clean water, landfill waste, deforestation, and air pollution and ultimately will help reduce the environmental effects of human activities. Due to a survey the market size of IoT will have a notice increase all over the world as all leading companies believe in the important role of IoT for both human life and the environment. Figure 3 represents a statically analysis for IoT platform market size from 2017 to 2023. To make better illustration about IoT effectiveness in combating climate change, next sub-sections will explain many life fields that IoT presents useful solutions for it to save the environment and leading to solve the problem of climate change.

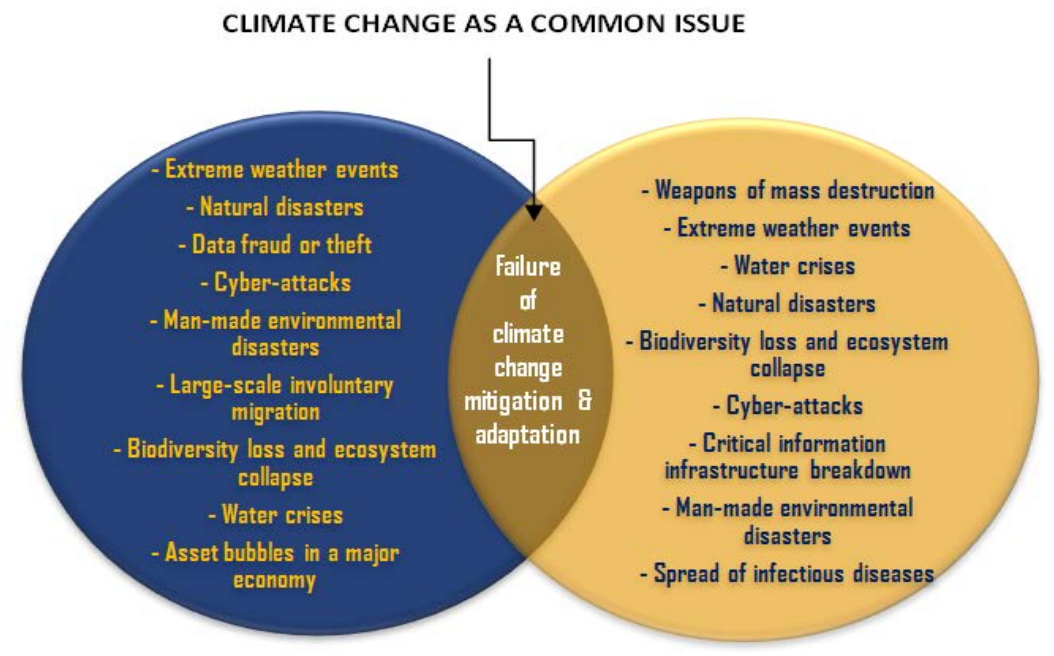

Figure 2. Intersection of Likelihood and Impact classifications of 2019

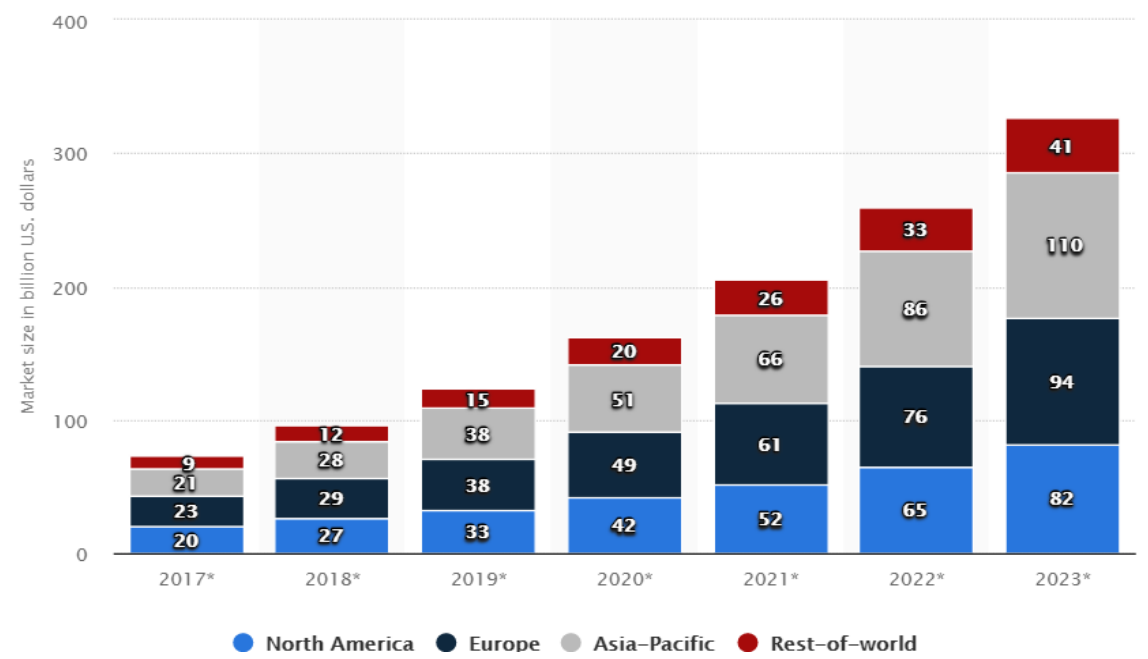

Figure 3. Global smart systems, services and IoT platform market size from 2017 to 2023, by region (in billion U.S. dollars) (Source: www.statista.com) 


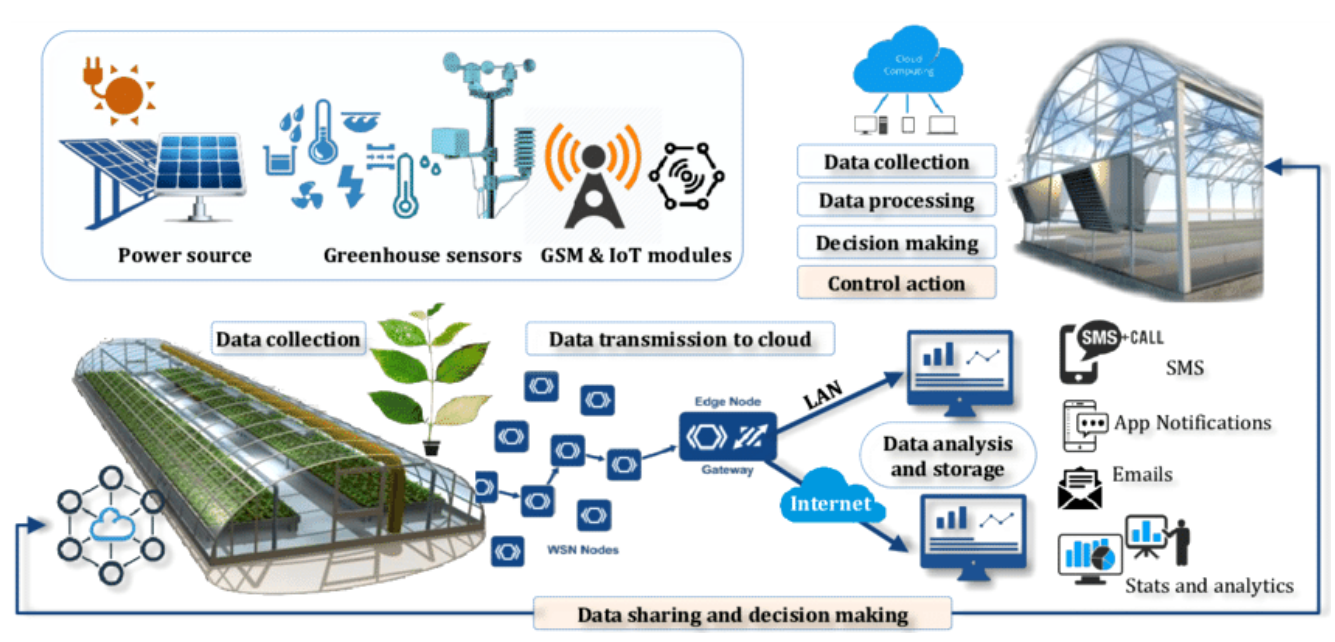

Figure 4. General components of a greenhouse environmental monitoring based in wireless sensor network and IoT concept (Source: R Shamshiri, Redmond, et al. (2018) [6])

\subsection{Agriculture}

Agriculture enterprises struggled many years to reduce their own impact on climate change through carbon emissions, as its carbon emissions are some of the largest on the planet. The agricultural sector is also one of the most important and, therefore, is looking to the Internet of Things for ways in which it can significantly improve its sustainability through schemes such as smart farming and precision agriculture. Deploying sensors to collect, process and transmit data in agricultural activities will lead to the development of advanced techniques in precision agriculture. IoT devices will also increase weather forecasting processes reliability which enabling farmers to make more efficient use of their resources and decrease wastes. Thus will improve the living conditions of low-income and developing countries, and also reducing the agricultural activities' environmental footprint. The International Telecommunication Union (ITU), found that the number of households living in remote rural areas with electricity and internet access is constantly increasing and this leads to strengthen the opportunity of involving technology in agriculture in many ways which limits the use of insecticides, fertilizer and water to the very minimum, as well as weather forecasting systems. Figure 4 explains the general components of a greenhouse environmental monitoring based in WSNs and IoT.

\subsection{Energy Storage and Building Automation}

Consumption of energy by societies is considered to be one of the main factors that are accelerating climate change. This is due to the use central heating or power plants generate a large amount of pollution, such as $\mathrm{CO}_{2}$ emissions. It must be understood that a large part of energy consumption is not caused only by our needs, but also by an inefficient management with little foresight. Actually, we consume more than what we really need because of too little data analysis. Monitoring people energy consumption makes it possible to reduce wastes. For instance, Nest, Honeywell, Lenox thermostat, While an unprogrammed thermostat can waste $20 \%$ of heating and cooling, they tackles the issue with a smart thermostat that learns your patterns and automatically adjusts to save energy, Figure 5 includes many smart thermostats examples. We can see that IoT save energy and carbon footprints with things as simple as using an app to turn off the lights or with apps like IFTTT, which hooks up to many different types of systems. IoT-enabled devices can be programmed, monitored, and controlled by an intelligent energy storage system so that they only work and consume power when necessary.

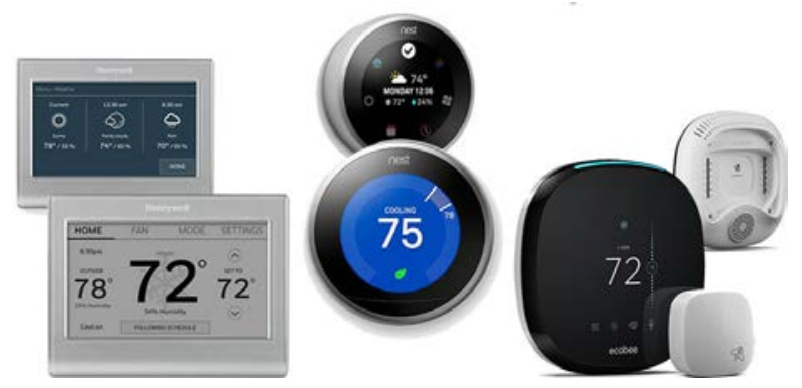

Figure 5. Smart Thermostats (Source: bestsmartthermostatreviews.com)

\subsection{Climate \& Environmental Related Data}

Climate models are critical for the process of evaluating the global climate change effect, but due to the complexity of our planet's weather systems means today's best models are still lacking and more data is still needed to model the climate and predict changes accurately. Since IoT paradigm is great for generating information, it will be the perfect solution to collect data about climate. There is already temperature, humidity and precipitation sensors around the world, but IoT devices can provide more robust data from even more sources. Such devices can also monitor ocean temperatures and sea levels more precisely than ever before, and this data will be very helpful for mitigating potential changes caused by climate change. Precisions from IoT devices will let scientists around the world detect minute changes and adjust their models accordingly. Since, the environment is always something that majority of people are passion about in some way or another, there are a huge number of projects that have been set up with the aim to cut emissions, pollution, illegal logging, and various other environmentally damaging or polluting activities. As their availability has become more widespread, IoT devices have been adopted by these 
causes to great effect. One example involves efforts to reduce deforestation, which accounts for $15 \%$ of all global carbon emissions, using sensors attached to trees that listen out to detect then report illegal logging. Another example involves a similar set up but being used to detect illegal poaching. This data can be seen in real-time and it is believed these kinds of technologies will enable groups to negotiate increased protections for these areas to help combat climate change and deforestation. Another very interesting example is to see how IoT fight with the pollution. Next figure presents applications of environmental uses of IoT.

\begin{tabular}{|c|}
\hline Smart Environment \\
\hline Real-World Deployments \\
\hline Natural Disaster Communication \\
\hline Endangered Species Protection \\
\hline Water System Monitoring \\
\hline Residential Community Networks \\
\hline Green Stream Technologies \\
\hline Connecting the Cook Islands \\
\hline Smart Flood Monitoring \\
\hline Radiation Leak Detection \\
\hline Air Pollution Monitoring \\
\hline
\end{tabular}

Figure 6. IoT Environmental Applications

\subsection{Smart Cities}

The use of connected devices will change how the humankind get engage with the environment, especially in urban ones which is where $70 \%$ of our species is projected to live by 2050 according to the latest revision of the UN population prospects. Smart buildings and cities are the next step in connecting buildings, homes, offices, warehouses, and all manner of public infrastructure. Operations such as lighting, power, waste collection, and alarm systems, as well as many other technologies, can be connected to centralized management systems that run them as efficiently as possible in order to reduce energy waste and the building's carbon footprint. Using IoT-powered intelligent transportation systems, a smart city could one day navigate you through its streets on a route that best avoids lingering on traffic lights or heavily congested areas. These systems would also be powered by the data they and other connected systems generated and shared that would then likely be utilized by a central management system in order to make the smart city truly intelligent. As climate change becomes increasingly relevant for everyone on the planet, the applications of IoT technologies listed above could all be utilized in order to help combat the effects of climate change and keep any extreme weather or environments to a minimum.

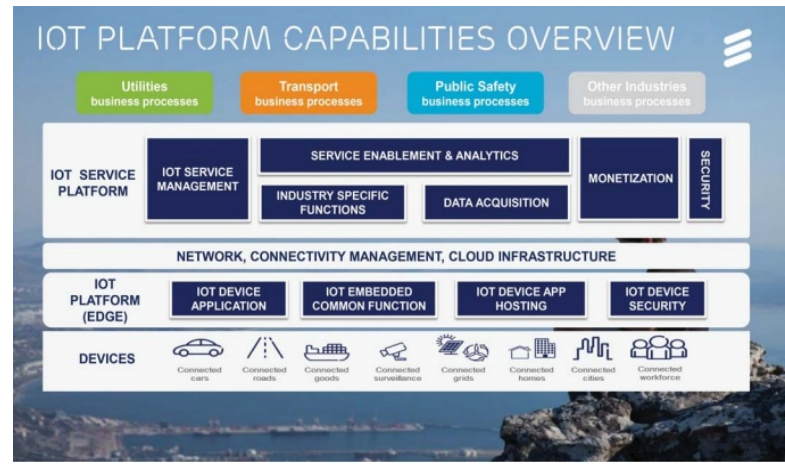

Figure 7. Smart Cities vision by Ericsson (Source: Let's look at some issues of debate in the IoT community. The rise of LPWAN is animating the industry, what does Ericsson think about LPWAN and forms of connectivity in general?)

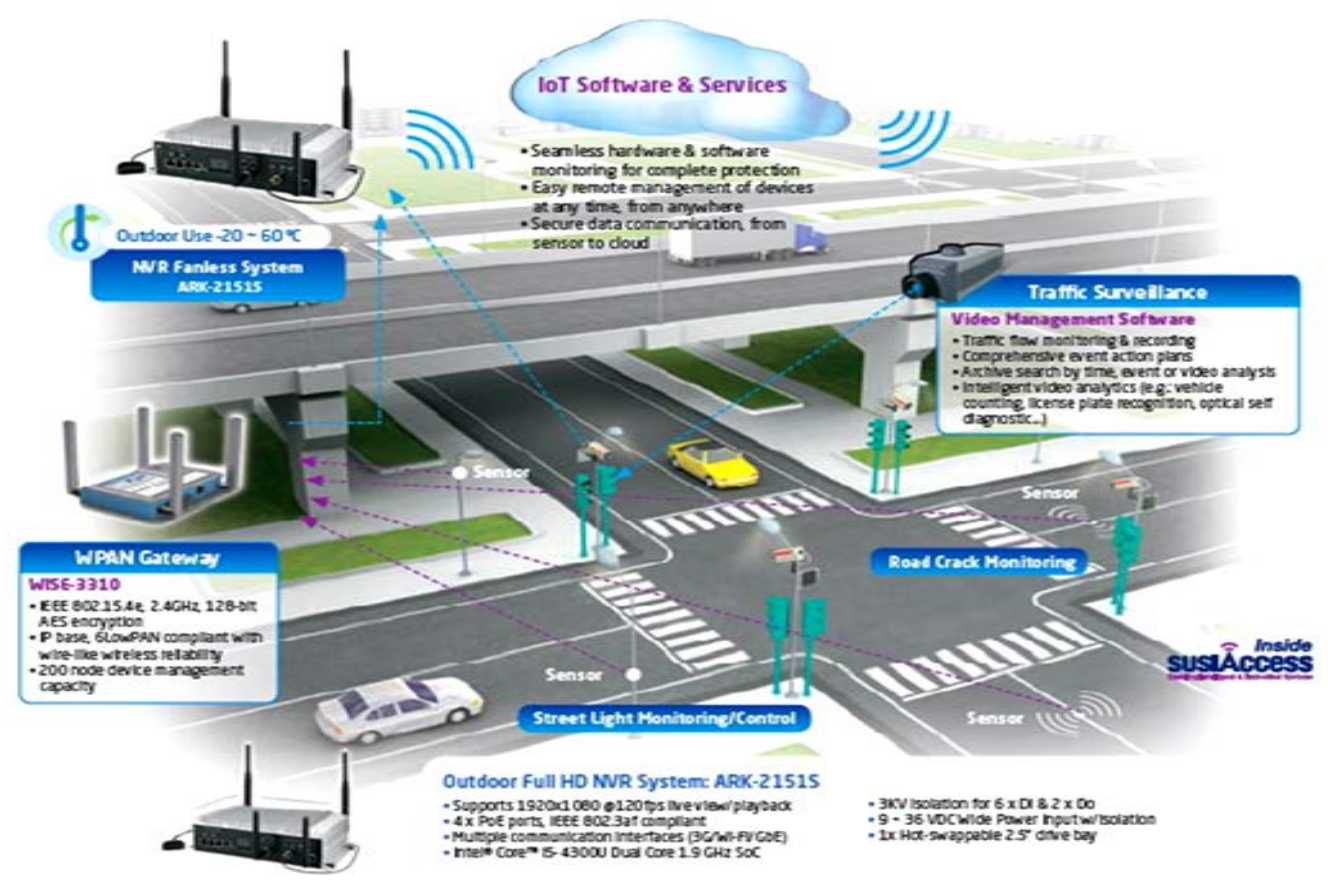

Figure 8. Intelligent Highways of ADVANTECH Project (Source: www.advantech.net.au) 


\subsection{Traffic and Transportation}

Using IoT-powered apps leads the world to reduce carbon dioxide emissions from cars through helping drivers find parking spaces faster and avoid congested roads to reduce the amount of time they spend driving. It also let drivers avoid traffic jams and schedule their trips when the roads are clear, which help in reducing fuel usage. For Instance, A project in Los Angeles uses IoT technology to synchronize traffic lights to enable traffic to flow more smoothly which lowered greenhouse gas emissions and saved more than 35 million gallons of gasoline annually. Also, IoT- trash cans have been implemented to notify collectors when they're full to make garbage collection working efficiently and thus reduce carbon emissions. Another profit that IoT gathered data let governments and transportation companies eliminate the unneeded routes and save operating costs and also reducing their carbon emissions. Furthermore, data can help develop better routes, helping public transportation entities gain new customers while giving people a greener alternative to less efficient personal vehicles as stop-and-go traffic leads to inefficient energy consumption. For instance, Intelligent Highways Project of advantech [7] which is explained in detail in Figure 8.

\subsection{Preventing Illegal Logging and Deforestation}

Nowadays, IoT-enabled devices are used widely to detect and report illegal logging and deforestation activities that contribute to climate change. Deforestation is considered to be one of the major causes of climate change. Due to a study, there is a need to implant a one billion of trees per a year to recover, which can only be possible if an automated system is used. IoT and drones are implemented, assembled and deployed to perform the process of deforestation monitoring efficiently. This reduces manpower requirements, time, cost and help in engaging modern agriculture techniques in planting and maintenance. For instance, Rainforest Connection project in a San Francisco is a start-up that transforms recycled cell phones into solar-powered listening devices which are attached to trees monitoring and detecting any illegal logging activities at great distance. Thus leading to enhance and increase the protection of deforestation-prone areas such as the Kalimantan region in Indonesia or the Amazon forest.

\subsection{Utilities}

IoT has a great role in helping different utilities as it allows them to use a fast responsive energy network that engages in predictive analytics to match and achieve energy generation with demand and this leads to store excess energy instead of wasting it. Also, smart meters can collect information about every building's energy usage and send that data back to utilities to help them with load balancing which will result in a waste reduction as well. All of this can lower the amount of fossil fuels utilities need to use to generate electricity and thus leads to reduce their carbon footprint.

\subsection{Waste Management}

The early predictions about IoT technology were that it would increase e-waste, but this problem was a part of the solution. IoT now provides a great way to manage wastes and plays a great role in the recycling process. The installed sensors would be able to inform consumers when it is ready to repair or recycle. They also notify the users which component can be reused so that they do not go trash early. For other wastage management, IoT products can be designed to automate disposal system timely.

\section{Case Study: Ericsson's IoT Environmental Technology Project}

Ericsson Research projects that what they call the SMART GRID in Figure 9 could alone cut greenhouse gas emissions by 3.9 percent by 2030 [5]. This developing IoT enabled energy supply network can detect and adjust for local changes in energy use and also provides great green benefits. One small component of this smart grid, the smart electricity meter, enables real-time two-way communication between utility and consumer, making it easier for the latter to meet energy demand with less waste through coordinating energy conservation and energy generation as well.

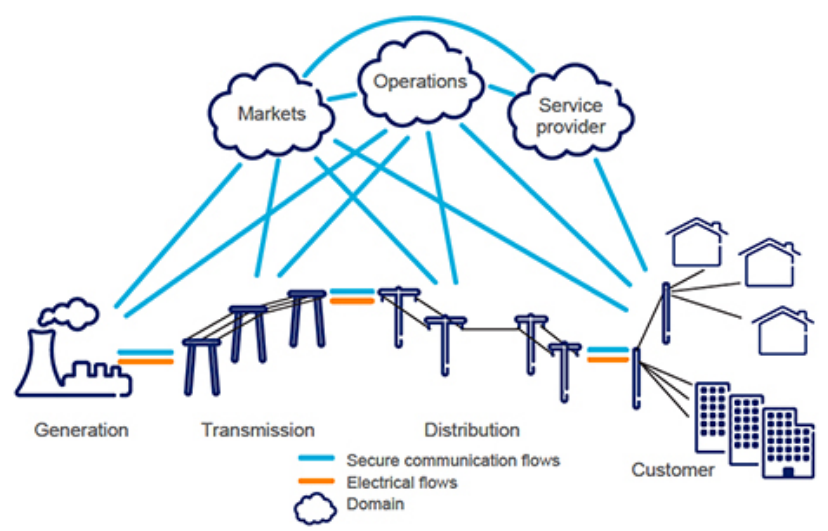

Figure 9. Ericsson Smart Grid ( Source: www.ericsson.com)

This will lead to motivating consumers for using less energy during rush hours. For example, In order to reduce the impact of carbon in daily economic activity, the World Green Building Council is working hard on coordinating local efforts to ensure that all new buildings operate at zero carbon. Sensors of IoT which are available today support all of the Council's main goals, including reducing energy consumption, generating renewable energy, and performing closed-loop measurement of carbon consumption and waste. Due to Ericsson Project, IoT-powered smart services could account for 3\% carbon reduction through increasing efficiency and reliance on disposable materials elimination in both the public and private sector. The Low-cost, low-power IoT devices bring smart agriculture (which minimizes use of fertilizer, insecticide, and water) to modernizing countries much more quickly than has been possible up to now.

According to Ericsson Research also, Smart agriculture could facilitate another 3\% reduction. Smart agriculture efforts including better cow monitoring that help in 
identifying ailing livestock based on the animal's behavior and position and this help also to screen, treat, and remove sick animals. This process will help the world to have improved meat and dairy yields and reduced over-treatment with antibiotics, as well as creating opportunities to reduce greenhouse gas emissions from unfit animals.

Ericsson Project also shows that the adoption of IoT monitoring and rerouting for oceanic cargo shipping is a possible way to reduce fuel consumption by up to $15 \%$. Early preventative maintenance help in avoiding five-week overhauls in favor of on-the-spot repairs could also reduce the need to build redundant fleets.

Due to the UN's climate change report [8], William Nordhaus and Paul Romer were awarded a Nobel Prize for their work on the economics of global warming. Their advice to world leaders and policymakers is to enact a tax to correspond to the social cost of carbon emissions by humankind. Unless, The world will be facing food and water shortages, disease, flooding, erosion and extreme political unrest in the next few decades. Though this type of aggressive policy change is currently viewed as political poison in the US, many major world economies (including China, Canada and parts of Europe) are already starting to implement carbon pricing. Romer states:

"People will see that there's a big profit to be made from figuring out ways to supply energy where they can do it without incurring the tax."

And that's where IoT solutions come into lead. In a 2015 report by Ericsson, researchers found that ICT could help reduce greenhouse gas emissions by up to 63.5 gigatons, or 15\%, across all industrial sectors by 2030 . Ericsson's examples of IoT solutions include the following:

- Electricity distribution - Small-scale renewable energy sources, smart metering, and smart grid solutions in households and buildings will help reduce energy consumption and cut losses.

- Services and industry - Smart solutions in the fields of government, healthcare and education will decrease the costs of service industries and promote socioeconomic development in underserved parts of the world.

- Transport - Smart travel solutions will help in getting lower emissions ratios by optimizing routes through traffic, find parking spaces quickly, and supporting a shift towards more eco-friendly alternatives, like public transport.

That's just the tip of the iceberg. By financially disincentivizing carbon usage, the world will see a numerous series of creative new ways to reduce waste and achieving lower greenhouse gas emissions across every industry. With efficiency and amazing power of IoT, there's no doubt it'll be a great ally to the fight. All of this means avoiding years and years of wasteful overconsumption of fuel, water and soil additives.

\section{Future is for Industrial Internet of Things (IIOT)}

A key reason for adopting IIoT by manufacturers, utility companies, agriculture producers and healthcare providers is to increase productivity and efficiency through smart and remote management and as well as being environment friendly and living in homogeneity. For example, Thames Water [9], the largest provider of drinking and waste-water services in the UK, is using sensors, and real-time data acquisition and analytics to anticipate equipment failures and provide fast response to critical situations, such as leaks or adverse weather events. The utility firm has already installed more than 100,000 smart meters in London, and it aims to cover all customers with smart meters by 2030. With 4,200 leaks detected on customer pipes, this program has already saved an estimated 930,000 liters of water per day for London. As another example, the deployment of 800 HART devices for real-time process management at Mitsubishi chemical plant in Kashima, Japan has been increasing the production performance by saving US\$20-30,000 per day that also averted a \$3million shutdown [10]. Agriculture controlled by IIoT can help farmers better in measuring different agricultural variables such as soil nutrients, fertilizer used, seeds planted, soil water, and temperature of stored produce, allowing to monitor down to the square foot through a dense sensor deployment, thereby almost doubling the productivity $[11,12,13]$. Companies like Microsoft (Farm- Beats project [14,15]); Climate Corp [16], AT\&T [17], and Monsanto [18] are promoting agricultural IoT. IIoT can also significantly impact the healthcare field. In hospitals, human or technological errors caused by false alarms, slow response, and inaccurate information are still a major reason of preventable death and patient suffering. By connecting distributed medical devices using IIoT technologies, hospitals can significantly overcome such limitations, thereby improving patient safety and experiences, and more efficiently using the resources. IIoT also provides opportunities to enhance efficiency, safety, and working conditions for workers. For example, using unmanned aerial vehicles (UAVs) allows inspecting oil pipelines, monitoring food safety using sensors, and minimizing workers' exposure to noise, and hazardous gases or chemicals in industrial environments. For example, Schlumberger is now use monitoring subsea conditions with unmanned marine vehicles travelling across oceans and collecting data for up to a year without fuel consumption or crew, moving under power generated from wave energy [19]. Through remote monitoring and sensing powered by IoT, mining industries can dramatically decrease safety-related incidents, while making mining in harsh locations more economical and productive. For example, Rio Tinto, a leading mining company, intends its automated operations in Australia to preview a more efficient future for all of its mines to reduce the need for human miners [20]. IIoT is a technology which not only enhances businesses but is also an eco friendly technology which benefits the environment and mankind as whole.

\subsection{IIoT Challenges}

As the future is for IIOT, there's a must to invest more in solving the main challenges that face it today. If these challenges are solved it will be a step forward saving the world and the environment. Energy Efficiency, Real Time Performance, Coexistence and Interoperability, Security and Privacy. The following figure summarizes the recent challenges of IIoT. 


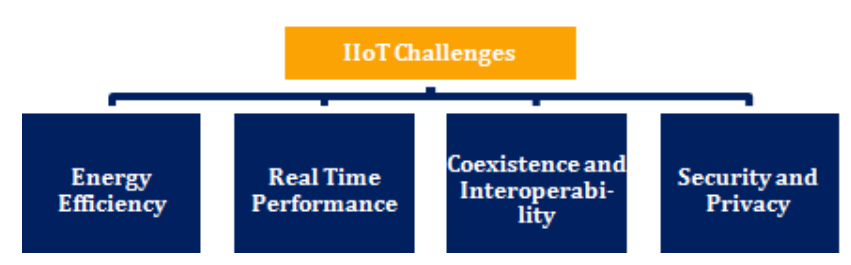

Figure 10. IIoT Challenges

\subsubsection{Energy Efficiency}

Practically, energy can be harvested from environmental sources, namely, thermal, solar, vibration, and wireless radiofrequency (RF) energy sources. Harvesting from such environmental sources is dependent on the presence of the corresponding energy source. Many energy efficient schemes for wireless sensor network (WSN) have been proposed in recent years, but those approaches are not immediately applicable to IIoT. IIoT applications typically need a dense deployment of numerous devices. Sensed data can be sent in queried form or in a continuous form which in a dense deployment can consume a significant amount of energy. Green networking is thus crucial in IIoT to reduce power consumption and operational costs. It will lessen pollution and emissions and make the most of surveillance and environmental conservation. LPWAN IoT technologies achieve low-power operation using several energy-efficient design approaches [21,22].

- A star topology, which eliminates the energy consumed through packet routing in multi-hop networks.

- Keep the node design simple by offloading the complexities to the gateway.

- Use narrowband channels, thereby decreasing the noise level and extending the transmission range.

- Lightweight communication protocols.

- Adopting low-power radio transceivers as described above.

\subsubsection{Real-Time Performance}

IIoT devices are typically deployed in noisy environments for supporting mission- and safety-critical applications, and have stringent timing and reliability requirements on timely collection of environmental data and proper delivery of control decisions. The QoS offered by IIoT is thus often measured by how well it satisfies the end-to-end (e2e) deadlines of the real-time sensing and control tasks executed in the system. Time-slotted packet scheduling in IIoT plays a critical role in achieving the desired QoS. The explosive growth of IIoT applications especially in terms of their scale and complexity has dramatically increased the level of difficulty in ensuring the desired real-time performance [23,24]. The fact that most IIoT must deal with unexpected disturbances further aggravate the problem. Unexpected disturbances can be classified into external disturbances from the environment being monitored and controlled (e.g., detection of an emergency, sudden pressure or temperature changes) and internal disturbances within the network infrastructure (e.g., link failure due to multi-user interference or weather related changes in channel SNR). In response to various internal disturbances, many centralized scheduling approaches [25] have been proposed. There are also a few works on adapting to external disturbances in critical control system. Hence, most of them have limited scalability. The concept of distributed resource management is not new.

In fact, distributed approaches have been investigated fairly well in the wireless network community (e.g., $[26,27])$. However, these studies typically are not concerned with real-time e2e constraints. Ensuring bounded response time to handle concurrent disturbances is still an open problem.

\subsubsection{Coexistence and Interoperability}

With the rapid growth of IIoT connectivity, there will be many coexisting devices deployed in close proximity in the limited spectrum. This brings forth the imminent challenge of coexistence in the crowded ISM bands. Thus, interference between devices must be handled to keep them operational. Existing and near future IIoT devices will most likely have limited memory and intelligence to combat interference or keep it to a minimum. Technologyspecific features of each IIoT technology may introduce additional challenges. To ensure good coexistence it will become important that future IIoT devices can detect, classify and mitigate external interference. Recently, some work regarding classifying interference via spectrum sensing [28] on IIoT devices has been presented but most of the existing work fails since a very long sampling window is needed as existing solutions use much more memory than what is available in existing IIoT devices. If the research of error correcting codes for IIoT devices should be successful, it is also important that more emphasis be given on investigating and understanding the complex radio environment where many of these IIoT devices will be deployed [29]. The challenges of device diversity in IIoT can be addressed along three dimensions: multimode radios, software flexibility, cross-technologycommunication [30].

\subsubsection{Security and Privacy}

Security is another critical concern in IIoT. In general, IIoT is a resource-constrained communication network which largely relies on low-bandwidth channels for communication among lightweight devices regarding CPU, memory and energy consumption. For this reason, traditional protection mechanisms are not sufficient to secure the complex IIoT systems, such as secure protocols, lightweight cryptography and privacy assurance. To secure the IIoT infrastructure, existing encryption techniques from industrial WSNs may be reviewed before applied to build IIoT secure protocols. For instance, scarce computing and memory resources prevent the use of resource-demanding crypto-primitives, e.g. Public-Key Cryptography (PKC). This challenge is more critical in the applications of massive data exchanged with real-time requirements. To address privacy and security threats in IIoT, one can argue for a holistic approach as pointed out in [31,32].

This means that aspects such as platform security, secure engineering, security management, identity management and industrial rights management must be taken into account, throughout the whole life cycle of the systems and products. There exist several security properties to consider when designing secure IIoT infrastructure [33]: 
1) IIoT devices need to be tamper resistant against potential physical attacks, such as unauthorized re-programming and passive secret stealing while allowing the authorized.

Users to update the security firmware on the device.

2) The storage of IIoT device should be protected against adversary by keeping the data encrypted to keep the confidentiality.

3) The communication network among the IIoT devices should be secured to keep confidentiality and integrity.

4) The IIoT infrastructure needs efficient identification and authorization mechanisms, so that only authorized entities can access the IIoT resource.

5) The system should be available within normal operation, even with the physical damage to the devices by malicious users. This guarantees the robustness of IIoT.

Typically, symmetric-key cryptography can provide a lightweight solution for IIoT devices. However, both the key storage and the key management are big issues if using symmetric-key encryption, especially when considering low capacity devices. Additionally, if one device in IIoT is compromised, it may leak all other keys. Public-key cryptography generally provides more secure features, and low storage requirements, but suffers from high computational overhead due to complex encryption. Thus, reducing the overhead of complex security protocols for public-key cryptosystems remains a major challenge for IIoT security.

\section{Conclusion}

Nowadays IoT technology getting increase rapidly so, the world could quickly move to one numerous connected network available to all. Too many researchers around the globe are working so hard to propose a standardized environmental sensor network driven by IoT technology. Climate change management with recent technologies is just the first step. Both public and private partnerships will need to put hand on hand and work hard to grow the investing ratio in such solutions. IoT and IIoT will provide actionable climate data. It will also give a control to environmental wastes by flowing up of individuals, energy and nature. It will continue adapting, as researchers and technology developers reach new assent on the action we need to take to protect ourselves not only against rising temperatures and dangerous climate changes but also against cyber attacks.

\section{Acknowledgements}

We would like to thank our colleagues who provided insight, expertise and encourage that greatly assisted the research. We also feel grateful to Nile Higher Institute for Engineering \& Technology as this work was supported in part by it.

\section{References}

[1] Gartner for business solutions, https://www.gartner.com/en.

[2] Techonomy magazine 2017 edition, https://www.ifoldsflip.com/i/747123-techonomy-magazine2017edition/5?m4=.

[3] The Global Risks Report 2019, 14th Edition, In partnership with Marsh \& McLennan Companies and Zurich Insurance Group.

[4] World Economic Forum, https://www.weforum.org/.

[5] Ericsson SMART GRID Project, https://www.ericsson.com/.

[6] R Shamshiri, Redmond, et al. "Advances in greenhouse automation and controlled environment agriculture: A transition to plant factories and urban agriculture." (2018)'

[7] Adaantch solutions, https://www.advantech.net.au.

[8] UN's Climate Change Report, https://www.ipcc.ch/sr15/.

[9] J. P. Tomas, "Thames water rolls out smart meter project in london,” 2017,

https://wiprodigital.com/cases/progressive-metering-a-utilitysstrategic-move-into-predictive-planning/.

[10] http://en.hartcomm.org/hcp/tech/applications/applications success mitsubishi chemical.html.

[11] M. H. Almarshadi and S. M. Ismail, "Effects of precision irrigation on productivity and water use efficiency of alfalfa under different irrigation methods in arid climates,” Journal of Applied Sciences Research, vol. 7, no. 3, pp. 299-308, 2011.

[12] H.-J. Kim, K. A. Sudduth, and J. W. Hummel, "Soil macronutrient sensing for precision agriculture,” Journal of Environmental Monitoring, vol. 11, no. 10, pp. 1810-1824, 2009.

[13] N. D. Mueller, J. S. Gerber, M. Johnston, D. K. Ray, N. Ramankutty, and J. A. Foley, "Closing yield gaps through nutrient and water management,” Nature, vol. 490, no. 7419, pp. 254-257, 2012.

[14] D. Vasisht, Z. Kapetanovic, J. Won, X. Jin, R. Chandra, S. Sinha, A. Kapoor, M. Sudarshan, and S. Stratman, "Farmbeats: An iot platform for data-driven agriculture," in 14th USENIX Symp. on Net. Syst. Design and Implementation (NSDI), 2017, pp. 515-529.

[15] Microsoft, "FarmBeats: IoT for agriculture," https://www.microsoft.com/en-us/research/project/farmbeats- iotagriculture/.

[16] C. Corporation, "Data-driven agricultural decisions and insights to maximize every acre,” https://www.climate.com.

[17] AT\&T M2X, "Agriculture iot software as a service (saas)," https://m2x.att.com/iot/industry-solutions/iot-data/agriculture/.

[18] J. Hawn, “Agricultural iot promises to reshape farming," RCR Wireless News, November 2015,

https://www.rcrwireless.com/20151111/internet-ofthings/agricultural-internet-of-things-promises-to-reshapefarming-tag15.

[19] Schlumberger, "Schlumberger robotics services," http://www.slb.com/ services/additional/robotics services.aspx.

[20] T. Simonite, "Mining 24 hours a day with robots," MIT Technology Review, December 2016, https://www.technologyreview.com/s/603170/mining-24-hours-aday-with-robots/.

[21] Saifullah, Abusayeed, et al. "Low-Power Wide-Area Network Over White Spaces." IEEE/ACM Transactions on Networking 26.4 (2018): 1893-1906.

[22] A. Saifullah, M. Rahman, D. Ismail, C. Lu, R. Chandra, and J. Liu, "SNOW: Sensor network over white spaces," in The 14th ACM Conf. on Embedded Network Sensor Systems (SenSys), 2016, pp. 272-285.

[23] P. Ferrari, A. Flammini, E. Sisinni, D. Brando, and M. Rocha, "Delay estimation of industrial iot applications based on messaging protocols,” IEEE Transactions on Instrumentation and Measurement, pp. 1-12, 2018.

[24] T. Zheng, M. Gidlund, and J. Akerberg, "Wirarb: A new mac protocol for time critical industrial wireless sensor network applications,” IEEE Sensors Journal, vol. 16, no. 7, pp. 2127-2139, April 2016.

[25] F. Dobslaw, T. Zhang, and M. Gidlund, "End-to-end reliabilityaware scheduling for wireless sensor networks," IEEE Transactions on Industrial Informatics, vol. 12, no. 2, pp. 758-767, 2016.

[26] C. Lu, A. Saifullah, B. Li, M. Sha, H. Gonzalez, D. Gunatilaka, C. $\mathrm{Wu}, \mathrm{L}$. Nie, and Y. Chen, "Real-time wireless sensor-actuator networks for industrial cyber-physical systems," Proceedings of the IEEE, vol. 104, no. 5, pp. 1013-1024, 2016.

[27] T. Zhang, T. Gong, C. Gu, H. Ji, S. Han, Q. Deng, and $\mathrm{X}$. S. Hu,n"Distributed dynamic packet scheduling for handling disturbances in real-time wireless networks,” in IEEE 
Real-Time and Embed. Tech. and App. Symp. (RTAS), 2017, pp. 261-272.

[28] T. M. Chiwewe, C. F. Mbuya, and G. P. Hancke, "Using cognitive radio for interference-resistant industrial wireless sensor networks: An overview,” IEEE Transactions on Industrial Informatics, vol. 11, no. 6, pp. 1466-1481, 2015.

[29] D. Ismail, M. Rahman, and A. Saifullah, "Low-power wide-area networks: Opportunities, challenges, and directions," in Proceedings of the Workshop Program of the 19th International Conference on Distributed Computing and Networking, ser. Workshops ICDCN '18, 2018, pp. 8:1-8:6.

[30] Sigfox, "Sigfox - the global communications service provider for the internet of things (iot)," http://sigfox.com.

[31] A.-R. Sadeghi, C. Wachsmann, and M. Waidner, "Security and privacy challenges in industrial internet of things," in Proceedings of the 52nd annual design automation conference. ACM, 2015, p. 54.
[32] G. Baldini, T. Peirce, M. Botterman et al., "Iot governance, privacy and security issues,” Position paper, European Research Cluster on the Internet of Things, 2015.

[33] A. W. Atamli and A. Martin, "Threat-based security analysis for the internet of things," in International Workshop on Secure Internet of Things (SIoT). IEEE, 2014, pp. 35-43.

[34] Elon Musk at Code Conference 2016, https://www.tesla.com/2016shareholdermeeting.

[35] T. Olofsson, A. Ahlen, and M. Gidlund, "Modeling of the fading statistics of wireless sensor network channels in industrial environments," IEEE Transactions on Signal Processing, vol. 64, no. 12, pp. 3021-3034, 2016.

[36] L. Ascorti, S. Savazzi, G. Soatti, M. Nicoli, E. Sisinni, and S. Galimberti, "A wireless cloud network platform for industrial process automation: Critical data publishing and distributed sensing,” IEEE Transactions on Instrumentation and Measurement, vol. 66, no. 4, pp. 592-603, 2017.

(C) The Author(s) 2019. This article is an open access article distributed under the terms and conditions of the Creative Commons Attribution (CC BY) license (http://creativecommons.org/licenses/by/4.0/). 